\title{
ESTADO NUTRICIONAL E CONSUMO ALIMENTAR DE USUÁRIOS DE CRACK DE UM MUNICÍPIO DO LITORAL NORTE DO ESTADO DO RIO GRANDE DO SUL
}

\section{Nutritional status and dietary intake of crack users in a city in the north coast} of the state of Rio Grande do Sul

Juliana Priscila Martins da Costa ${ }^{1}$, Majoriê de Freitas de Barros ${ }^{1}$, Ângela Martinha Bongiolo1, Ingrid Dalira Schweigert Perry ${ }^{1,2}$, Marco Antonio da Silva ${ }^{1}$

1Departamento de Nutrição, Unidade Acadêmica da Saúde, Universidade do Extremo Sul Catarinense - UNESC, Criciúma, SC.

2Programa de Pós Graduação em Saúde Coletiva (Mestrado Profissional), Unidade Acadêmica da Saúde - UNESC, Criciúma, SC.

\section{Endereço para Correspondência:}

Marco Antônio da Silva

Universidade do Extremo Sul Catarinense. Bairro Universitário. 88806-000

Criciúma - SC

Fone: +55 48 3431-2560 / Fax: +55 $483431-2736$

Email: mar@unesc.net 


\title{
Resumo
}

O objetivo do estudo foi avaliar o estado nutricional e o consumo alimentar de usuários de crack submetidos ou não a tratamento para dependência química. Trata-se de um estudo transversal com 40 usuários de crack com idade $\geq 18$ anos. Foram avaliados 0 peso/estatura para cálculo do Índice de Massa Corporal (IMC), o percentual de gordura corporal (\%GC), uso de substâncias psicoativas e consumo alimentar. Os participantes eram predominantemente do sexo masculino $(87,5 \%)$, poliusuários (97,5\%), com mais de cinco anos de consumo de crack e frequência de uso diário em $90 \%$ dos casos, com idade média de $32,7 \pm 8,1$ anos; $27,5 \%$ dos indivíduos apresentava-se em sobrepeso/obesidade segundo o IMC, havendo associação entre estado nutricional e estar ou não em algum tipo de tratamento $(p=0,016$; teste quiquadrado); 45,8\% dos indivíduos em tratamento apresentavam sobrepeso/obesidade, em contraposição a nenhum dos indivíduos do grupo sem tratamento); 56,3\% dos usuários sem tratamento apresentavam baixo \%GC; $62,5 \%$ do grupo em tratamento apresentavam alto \%GC, havendo associação entre o \%GC e estar ou não em tratamento ( $p=0,043$; teste qui-quadrado). Foi relatado consumo frequente de doces (4 a 7 vezes por semana) e pouco frequente de vegetais e frutas pelos grupos não internados. O sobrepeso/obesidade e maior \% de GC foi observado em indivíduos em tratamento e eutrofia/baixo peso e menor \% de GC em usuários sem tratamento. $A$ qualidade da alimentação não parece ser substancialmente diferenciada entre usuários em tratamento ou não.

Palavras-chave: Crack; Estado nutricional; Consumo Alimentar.

\begin{abstract}
Objective: To assess the nutritional status and dietary intake of crack users submitted or not to treatment for chemical dependency. Methods: Cross-sectional study with 40 crack users over 18 (that were not undergoing treatment or monitored in Psychosocial Care Center or admitted in Therapeutic Community). Weight and height to calculate Body Mass Index (BMI), body fat percentage (\%BF), substance use and food consumption were evaluated. Results: most of the users were male (87.5\%), poliusers (97.5\%) with over five years of crack use and frequency of daily consumption. The average age of participants was $32.7 \pm 8.1$ years;. $27.5 \%$ of subjects presented in
\end{abstract}




\section{Artigo Original}

\section{Atenção à Saúde}

overweight/obese according to BMI, with an association between nutritional status and whether or not some kind of treatment ( $p=0.016$, chi-square test); $45.8 \%$ of individuals in treatment were overweight/obese, as opposed to any of the individuals in the untreated group; $56.3 \%$ of users without treatment had low \%BF; $62.5 \%$ of the treatment groups had a high \%BF, with an association between the \% BF and whether or not in treatment ( $p=0.043$, chi-square test). Frequent consumption of sweets has been reported ( 4 to 7 times a week) and infrequent of vegetables and fruits by nonhospitalized groups. Conclusion: overweight / obesity and higher GC\% was observed in individuals undergoing treatment for chemical dependency and normal weight / low weight and lower GC\% in untreated users. The quality of food does not seem to be substantially different among users in treatment or not.

Keywords: Crack; Nutritional Status; Food Consumption.

\section{INTRODUÇÃO}

O abuso de drogas lícitas e ilícitas constitui um problema de saúde pública com grande impacto social, sendo o Brasil apontado como uma das nações emergentes onde o consumo de estimulantes como a cocaína, seja na forma intranasal ("pó") ou fumada (crack, merla ou oxi) é crescente ${ }^{1}$. Com início de ação rápida, de 5 a 10 segundos, os efeitos estimulantes do crack duram cerca de 5 minutos, levando o usuário a repetir compulsivamente o seu uso². Seus efeitos deletérios impactam em prejuízos orgânicos e psíquicos, além de exposição social relacionada ao uso $^{3}$.

A organização de ações efetivas de saúde que atendam esses usuários e que promovam reabilitação e promoção da saúde ${ }^{4}$, assim como possibilidade de rastreamento destes após alta como alternativa para localizar populações ocultas ${ }^{5}$, são de extrema importância. No enfrentamento ao uso do crack, diversas estratégias foram desenvolvidas e incorporadas ao Sistema Único de Saúde ${ }^{6}$, além de movimentos da própria sociedade instaurando clínicas e Comunidades Terapêuticas ${ }^{4}$.

O desenvolvimento do trabalho em equipe comprometida em conhecer e atender às necessidades sociais e de saúde dos usuários de crack e suas famílias torna-se elementar ${ }^{7}$, contudo nos espaços terapêuticos nem sempre as equipes 
Artigo Original

Atenção à Saúde

multidisciplinares incluem a preocupação com a reconhecida interferência do uso abusivo de substâncias psicoativas no comportamento alimentar ${ }^{8}$ e no perfil nutricional destes usuários, havendo relatos de reflexos importantes nestes aspectos, como, por exemplo, a migração de estado nutricional para sobrepeso em indivíduos em tratamento voluntário para a dependência química ${ }^{9}$. Adicionalmente, a intoxicação aguda pode produzir quadro de anorexia e trazer comprometimento do sistema digestivo, sendo comum o aparecimento de náuseas, vômitos e diarreia e, em alguns casos mais graves, o surgimento de úlceras no trato gastrointestinal ${ }^{10}$, situações que podem impactar negativamente no estado nutricional. Neste contexto este estudo visou avaliar o estado nutricional e consumo alimentar de usuários de crack que se encontravam ou não submetidos a tratamento para dependência química, em um município no litoral do estado do Rio Grande do Sul.

\section{MÉTODOS}

Estudo com delineamento transversal, de caráter descritivo, com dados coletados de janeiro a maio de 2014. A amostra de conveniência foi composta por 40 usuários de crack, todos maiores de 18 anos e residentes em um município do litoral norte do Rio Grande do Sul. Dos participantes, 16 usuários não estavam submetidos a tratamento para dependência química e foram recrutados por indicação de uma organização não governamental para dependentes químicos, 12 eram acompanhados pelo Centro de Atenção Psicossocial (CAPS) e 12 encontravam-se internados em Comunidade Terapêutica. Constituíram critérios de inclusão idade $\geq 18$ anos e histórico de consumo contínuo de crack no mínimo por 1 ano, sendo excluídos gestantes, usuários sob tutela judicial ou com a consciência visivelmente alterada pelo uso de drogas no momento da abordagem.

Foram coletados, por entrevistadores capacitados para tal, dados sociodemográficos (idade, sexo, escolaridade, renda e composição familiar), antropométricos (peso e estatura), de composição corporal (percentual de gordura corporal - \%GC), consumo de substâncias psicoativas (tempo e frequência de consumo de crack, tempo de abstinência, uso de outras substâncias, história familiar de consumo de substâncias e tratamento prévio) e consumo alimentar. 
A coleta das variáveis sociodemográficas e consumo de substâncias psicoativas foi realizada por meio de entrevista. Os dados antropométricos (peso e composição corporal) para avaliação do estado nutricional foram aferidos utilizando balança digital com capacidade de leitura por bioimpedância (Tanita $\AA$, Tanita Corporation, Tokyo, Japan - modelo Ironman com capacidade de $150 \mathrm{~kg}$ e $100 \mathrm{~g}$ de acurácia) e para aferição da estatura utilizou-se o estadiômetro vertical portátil em escala milimétrica de $2.000 \mathrm{~mm}$, com precisão de $1 \mathrm{~mm}$ (Sanny®), American Medical Brazil, SBC, SP). Para a obtenção dos dados de peso corpóreo e estatura os indivíduos foram orientados a ficar descalços, com roupas leves e inspirar no momento da aferição da estatura. Para determinação do Índice de Massa Corporal (IMC) foi utilizada a fórmula IMC= (peso[kg]/altura[m²], sendo os pontos de corte para a classificação do estado nutricional os estabelecidos pela Organização Mundial da Saúde ${ }^{11}$, classificando em baixo peso, eutrofia, sobrepeso/obesidade. O \%GC obtido por meio da bioimpedância foi classificado segundo Lohman et al. (1992) apud Heyward e Stolarczyk ${ }^{12} \mathrm{em}$ abaixo da média, média e acima da média.

Para avaliação do consumo alimentar dos usuários não internados (sem tratamento e frequentadores do CAPS), foi utilizado questionário de frequência de consumo alimentar (QFA) dos últimos seis meses, de acordo com metodologia de Fisberg et al. ${ }^{13}$, sendo, para efeito de análise descritiva, agrupados os alimentos ricos em amido, gorduras, frutas, verduras, outros alimentos ricos em carboidratos simples (doces em geral), leite e derivados e carnes. Tendo em vista que a alimentação segue um padrão de oferta aos usuários de crack internados em comunidade terapêutica, foi coletada a frequência da oferta dos grupos alimentares, baseado no QFA aplicado para os não internados, e posteriormente verificado o consumo destes grupos pelos participantes da pesquisa.

Foram seguidas as diretrizes e normas para pesquisa com seres humanos, traçadas pelo Conselho Nacional de Saúde, respeitando-se a resolução número 466/2012, tendo o protocolo sido aprovado pelo Comitê de Ética em Pesquisa da Universidade do Extremo Sul Catarinense, Criciúma, SC. A entrevista com os sujeitos do estudo teve caráter individual, voluntário e foi realizada mediante assinatura do Termo de Consentimento Livre e Esclarecido.

Os dados foram analisados utilizando o software SPSS versão 18.0. $\mathrm{Na}$ análise descritiva, foram apresentadas as frequências relativas e absolutas para as 
Artigo Original

Atenção à Saúde

variáveis categóricas, além de medidas de tendência central e de dispersão para as variáveis contínuas. Para fazer as associações entre as classificações do estado nutricional e os grupos com e sem tratamento foi utilizado o teste qui-quadrado, usando-se o nível de significância de 5\%.

\section{RESULTADOS}

A Tabela 1 apresenta a descrição da amostra estudada. No grupo estudado houve predominância de usuários do sexo masculino, escolaridade média completa ou incompleta, com renda própria e renda familiar de até três salários mínimos. A idade variou de 20 a 52 com média de $32,7 \pm 8,1$ anos.

Tabela 1. Indicadores Demográficos e Socioeconômicos de usuários $(n=40)$ de crack. Torres, Rio Grande do Sul, 2014.

\begin{tabular}{ccc}
\hline Indicadores & $\mathbf{n}$ & $\%$ \\
\hline Sexo & 5 & 12,5 \\
Feminino & 35 & 87,5 \\
Masculino & & \\
Escolaridade & 5 & 12,5 \\
Ensino Fundamental & & \\
$\quad$ Incompleto & 11 & 27,5 \\
Ensino Fundamental & 10 & 25 \\
Completo & 11 & 27,5 \\
Ensino Médio Incompleto & 3 & 7,5 \\
Ensino Médio Completo & & 70 \\
Ensino Superior Completo & 28 & 30 \\
\hline Possui Renda Própria & 12 & 23,5 \\
Sim & & 45 \\
Não & 9 &
\end{tabular}


Artigo Original

Atenção à Saúde

\begin{tabular}{ccc}
\hline \hline Indicadores & $\mathbf{n}$ & $\%$ \\
\hline 4 ou mais pessoas & 13 & 32,5 \\
Renda Familiar & & \\
Até 1 salário mínimo & 5 & 12,5 \\
$>1$ a 3 salários mínimos & 13 & 32,5 \\
$>$ 3 salários mínimos & 22 & 55 \\
\hline
\end{tabular}

A tabela 2 mostra o perfil dos usuários relativamente ao uso do crack. A maioria da população era usuária há mais de cinco anos, relatava uso diário de crack e era poliusuária, sendo que mais da metade já haviam estado em tratamento para dependência e estavam em abstinência.

Tabela 2. Aspectos relacionados ao uso de crack por seus usuários $(n=40)$. Torres, Rio Grande do Sul, 2014.

\begin{tabular}{ccc}
\hline Indicadores & $\mathbf{n}$ & $\%$ \\
\hline Tempo de uso de crack & 13 & 32,5 \\
Menos de 3 anos & 10 & 25 \\
De 3 a 5 anos & 17 & 42,5 \\
Mais de 5 anos & & \\
\hline Frequência de uso de & 36 & 90 \\
crack & 3 & 7,5 \\
Diário & 1 & 2,5 \\
Semanal & & \\
Mensal & 39 & 97,5 \\
Utilização de outras & 1 & 1,9 \\
drogas & & \\
Sim & 31 & 79,4 \\
Não & 35 & 89,7 \\
Álcool & 16 & 41 \\
Tabaco & & \\
Ansiolíticos &
\end{tabular}




\begin{tabular}{ccc}
\hline Indicadores & $\mathbf{n}$ & $\%$ \\
\hline Maconha & 28 & 71,8 \\
Cocaína & 30 & 76,9 \\
Solventes & 6 & 15,4 \\
Ecstasy & 5 & 12,8 \\
Anfetaminas & 3 & 7,7 \\
\hline Possui familiar usuário & & \\
de drogas & & 70 \\
Sim & 28 & 30 \\
Não & 12 &
\end{tabular}

\section{Grau de parentesco do}

familiar usuário de drogas

\begin{tabular}{ccc} 
Pai & 9 & 15,5 \\
Mãe & 0 & 0 \\
Irmãos & 18 & 64,5 \\
Filhos & 1 & 3,6 \\
Cônjuge & 6 & 21,4 \\
Outros & 6 & 21,4 \\
\hline
\end{tabular}

\section{Esteve em tratamento}

Sim $\quad 26 \quad 65$

Não $\quad 14 \quad 35$

\section{Tempo de abstinência}

$\begin{array}{ccc}\text { Em uso } & 16 & 41 \\ \leq 3 \text { meses } & 9 & 22,5 \\ \text { Mais de 3 meses } & 15 & 37,5\end{array}$

Segundo O IMC, $27,5 \%$ dos indivíduos apresentavam-se em sobrepeso/obesidade, observando-se uma associação entre estado nutricional e estar ou não em algum tipo de tratamento ( $p=0,016$; teste qui-quadrado). Aproximadamente a metade dos indivíduos em tratamento no CAPS e em comunidade terapêutica apresentavam sobrepeso/obesidade, em contraposição a nenhum dos indivíduos do grupo sem tratamento. A análise do \%GC não evidenciou usuários em risco de 
Artigo Original

Atenção à Saúde

doenças ou desordens associadas à desnutrição, contudo 32,5\% apresentavam o $\% G C$ abaixo da média e 42,5\% acima da média e/ou em risco de doenças ou desordens associadas à obesidade. Também neste parâmetro observou-se associação entre $0 \% G C$ e estar ou não em algum dos grupos de tratamento ( $p=0,043$; teste qui-quadrado) (Tabela 3). Quando analisados o grupo sem tratamento e os grupos em tratamento de forma conjunta, reiteram-se essas associações para ambos os parâmetros ( $p=0,013$ para o IMC; $p=0,003$ para o \%GC; teste qui-quadrado).

Tabela 3. Classificação do estado nutricional de usuários de crack segundo o Índice de Massa Corporal e Percentual de Gordura Corporal.

\begin{tabular}{cccccccccc}
\hline & $\begin{array}{c}\text { Total } \\
(\mathbf{n}=\mathbf{4 0})\end{array}$ & $\begin{array}{c}\text { Sem } \\
\text { tratamento } \\
(\mathbf{n}=\mathbf{1 6})\end{array}$ & $\begin{array}{c}\text { CAPS } \\
(\mathbf{n}=\mathbf{1 2})\end{array}$ & $\begin{array}{c}\text { Comunidade } \\
\text { Terapêutica } \\
(\mathbf{n}=\mathbf{1 2})\end{array}$ & $\mathbf{p}^{\star *}$ \\
& $\mathbf{n}$ & $\%$ & $\mathbf{n}$ & $\%$ & $\mathbf{n}$ & $\%$ & $\mathbf{N}$ & $\%$ & \\
\hline IMC & & & & & & & & & \\
Baixo peso & 4 & 10,0 & 3 & 18,7 & 1 & 8,3 & 0 & 0 & 0,016 \\
Eutrofia & 25 & 62,5 & 13 & 81,3 & 6 & 50,0 & 6 & 50,0 & \\
Sobrepeso/obesidade & 11 & 27,5 & 0 & 0 & 5 & 41,7 & 6 & 50,0 & \\
\%GC & & & & & & & & & \\
Abaixo da média & 13 & 32,5 & 9 & 56,3 & 2 & 16,7 & 2 & 16,7 & 0,043 \\
Média & 10 & 25,0 & 5 & 31,2 & 2 & 16,7 & 3 & 25.0 & \\
Acima da média* & 17 & 42,5 & 2 & 12,5 & 8 & 66,6 & 7 & 58,3 & \\
\hline
\end{tabular}

IMC= Índice de Massa Corporal (WHO,1997); \%GC= porcentagem de gordura corporal (Lohman, 1997) apud Heyward e Stolarczyk (2000) ${ }^{12}$; CAPS= Centro de Atendimento Psicossocial.

* Acima da média ou risco de doenças e desordens associadas a obesidade.

** Teste qui-quadrado.

De acordo com a análise de frequência alimentar, a maior parte dos usuários frequentadores de CAPS e dos que estavam sem tratamento relatou consumir doces em frequência elevada de dias da semana. Já as fontes proteicas de origem animal como as carnes e leite eram consumidas nessa frequência por menos de $50 \%$ dos usuários sem tratamento. Enquanto o comportamento se repetia no que se refere às carnes nos frequentadores de CAPS, o leite era consumido por número expressivamente superior destes usuários comparativamente aos sem tratamento. $O$ consumo de vegetais e de frutas era pouco frequente em ambos os grupos, 


\section{Artigo Original}

\section{Atenção à Saúde}

ressaltando-se que no grupo sem tratamento uma frequência expressiva de usuários raramente ou nunca consumiam frutas (Figuras 1 e 2). A oferta de grupos alimentares aos usuários internados em Comunidade Terapêutica era diária no que se referem aos amidos, carnes, leites e gorduras, enquanto que a oferta de vegetais e de doces era de três vezes por semana e frutas eram raramente oferecidas.

Figura 1. Frequência de consumo de grupos de alimentos por usuários de crack sem tratamento.

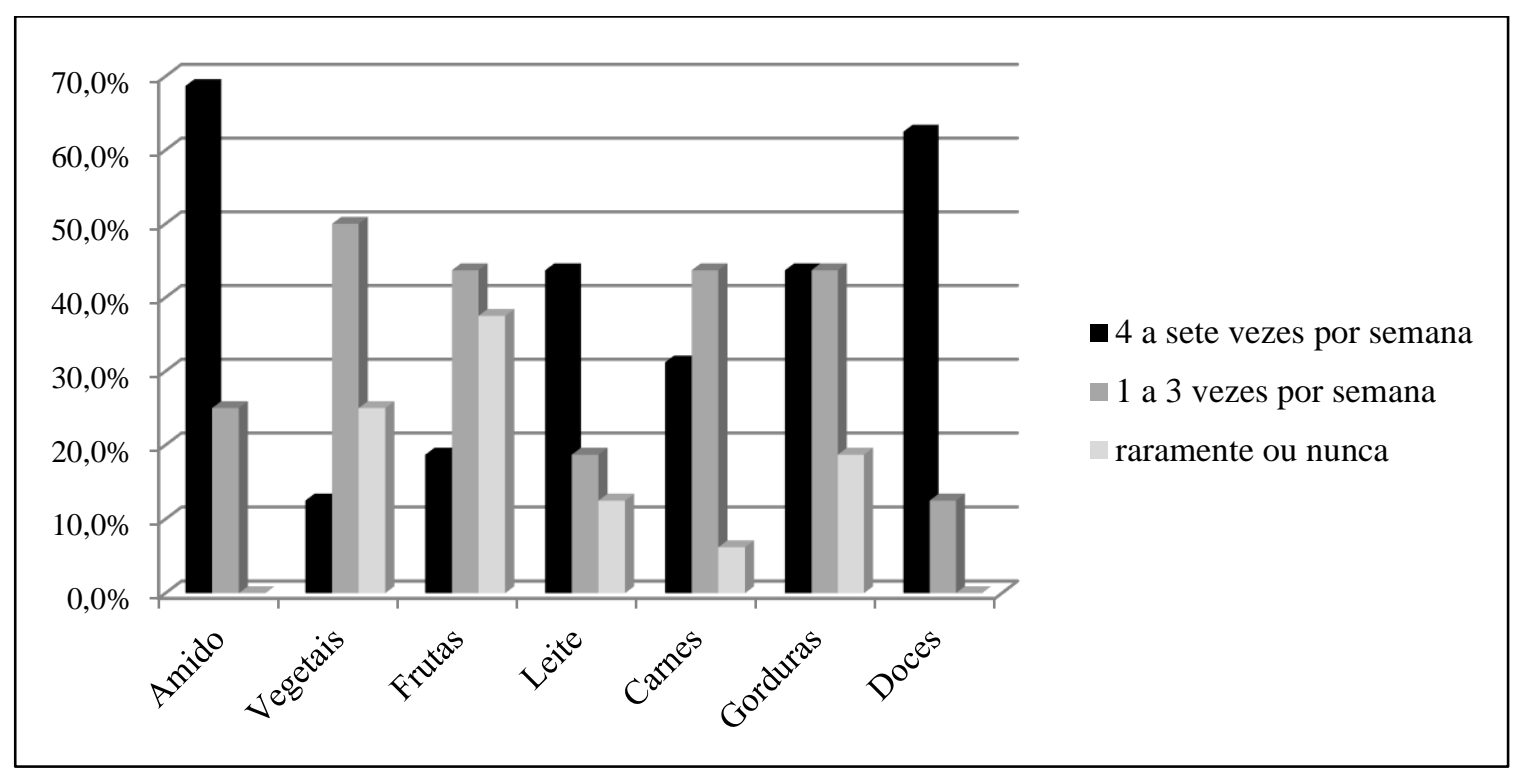

Figura 2. Frequência de consumo de grupos de alimentos por usuários do crack frequentadores de Centros de Atendimento Psicossocial (CAPS).

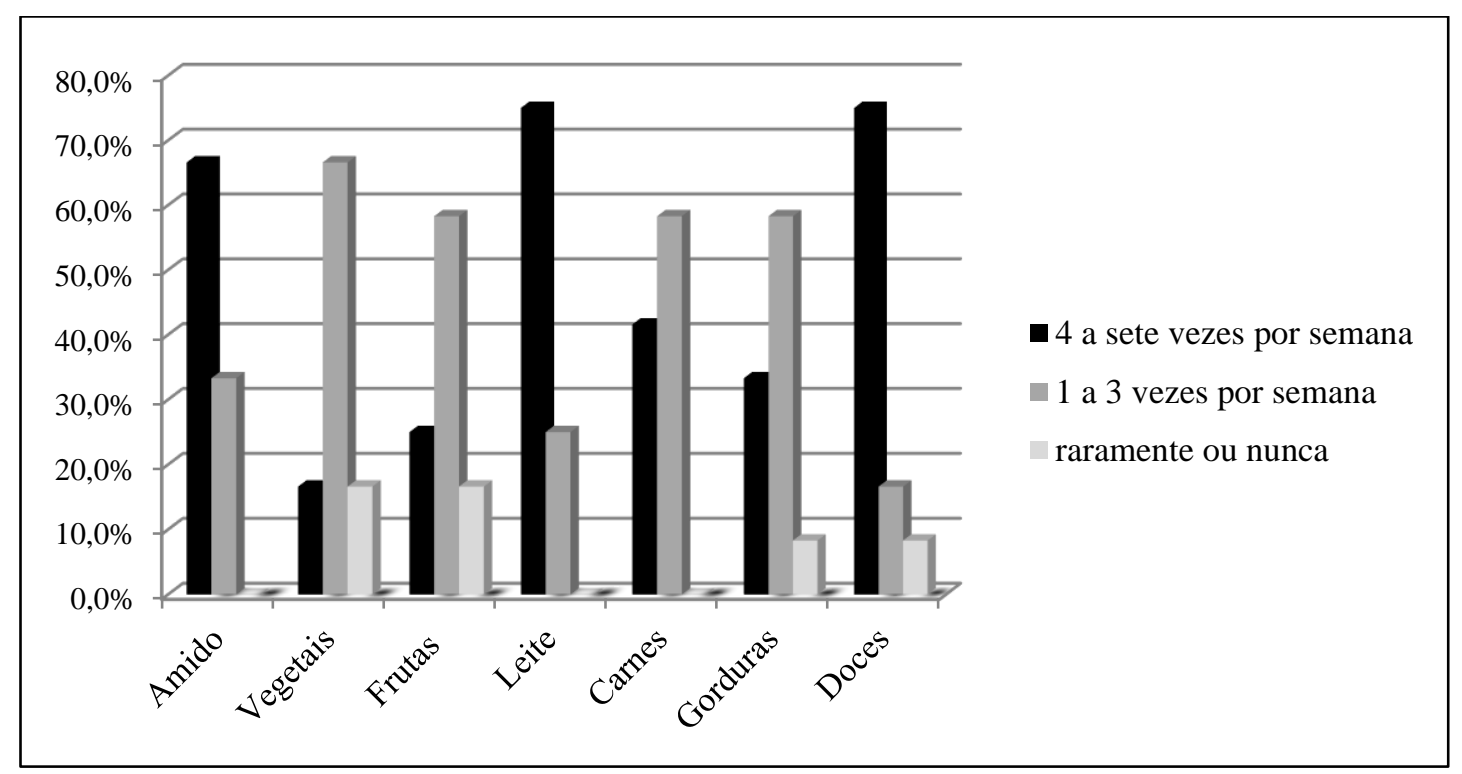




\section{DISCUSSÃO}

Os principais achados do presente estudo apontam cerca de metade dos indivíduos em tratamento (CAPS ou comunidade terapêutica) em sobrepeso/obesidade segundo o $\mathrm{IMC}$, em contraposição aos indivíduos que não estavam em tratamento, no quais não foi observado sobrepeso/obesidade. A elevada prevalência (mais de 60\%) de indivíduos em tratamento com percentual de gordura corporal elevado reitera esses achados, identificando, contudo, indivíduos com gordura corporal abaixo da média, especialmente nos usuários sem tratamento. Tanto usuários frequentadores de CAPS quanto os que estavam sem tratamento relataram consumir doces em frequência elevada de dias da semana; fontes proteicas de origem animal (carnes) eram consumidas nessa frequência por menos de $50 \%$ dos usuários sem tratamento e frequentadores de CAPS, contudo, o leite era consumido por número expressivamente superior destes usuários comparativamente aos sem tratamento; o consumo de vegetais e de frutas era pouco frequente em ambos os grupos, sendo também pouco oferecida aos indivíduos em comunidade terapêutica.

As características sociodemográficas dos indivíduos estudados assemelhamse em alguns aspectos de outros estudos, tais como a predominância de usuários jovens e do sexo masculino ${ }^{14,15,16}$ e baixo nível socioeconômico ${ }^{4}$. No que se refere à escolaridade, diferentemente do estudo de Araújo Silva ${ }^{14}$, que evidencia predominância de usuários tratados em CAPSad com fundamental incompleto $(46,2 \%)$, no presente estudo há maior concentração de usuários com ensino fundamental completo e médio completo, não havendo relato de analfabetismo.

O uso de outras drogas ao longo da vida referido por $97,5 \%$ dos entrevistados retrata o encontrado também por outros estudos que igualmente apontam que, em sua maioria, os indivíduos são poliusuários de drogas ${ }^{9,17}$. O longo período de tempo de uso evidenciado no presente estudo, assim como o consumo diário relatado e tratamentos prévios denotam o alto poder de dependência que o crack proporciona ao usuário. Apesar de percentual importante de usuários avaliados que estavam em acompanhamento terapêutico relatarem abstinência da droga há mais de três meses, percentual expressivo informou estar há menos de três meses sem consumir crack. Estudo com usuários internados relata que $80 \%$ já haviam tentado parar de fumar crack pelo menos alguma vez (em média 3,7 vezes) e que $60 \%$ fizeram algum tipo de 
Artigo Original

Atenção à Saúde

tratamento para interromper o uso da substância, sendo que o tipo de tratamento mais comum foi a internação para desintoxicação, seguido de tratamentos em clínicas e fazendas terapêuticas ${ }^{18}$. Em outro estudo, 72,7\% dos usuários também reportaram tratamentos prévios ${ }^{4}$.

Como esperado, usuários que não estavam em tratamento no momento da avaliação não apresentavam sobrepeso ou obesidade segundo o IMC. Corroborando com este resultado, mais de 50\% deles apresentavam \%GC abaixo da média, apontando para o fato de que o risco de desnutrição se faz presente nos usuários de crack em pleno uso da droga. No estudo de Etchepare et al. ${ }^{19}$, 95,4\% dos dependentes químicos relataram não sentir fome quando usavam a droga e 45,4\% sequer se alimentavam durante todo dia quando estavam sob o efeito da droga, o que poderia, dentre outros fatores, estar contribuindo para o déficit do estado nutricional.

Diferentemente do grupo sem tratamento, nos indivíduos em tratamento para dependência houve uma distribuição relativamente equitativa entre a eutrofia e excesso de peso. Ainda, considerando a adiposidade aferida por meio da bioimpedância, há resultados opostos no que diz respeito aos extremos de gordura corporal, excesso de gordura corporal e baixa gordura corporal, estando cerca de 60 $\%$ dos indivíduos sem tratamento abaixo do \%GC preconizado, e nos grupos em tratamento, cerca de 60\% acima do preconizado. Ou seja, nas modalidades terapêuticas avaliadas (CAPS e comunidade terapêutica) pode-se perceber maiores percentuais de usuários com sobrepeso/obesidade e \%GC acima da média e/ou risco de doenças ou desordens associadas à obesidade.

Estudos recentes evidenciaram também uma alteração importante de indicadores antropométricos em indivíduos internados para desintoxicação do crack. Há indicativos de IMC adequado em sua maioria, contudo, com baixo percentual de gordura corporal ${ }^{20}$, ou, ainda, alteração do \%GC e IMC durante a internação, levando a maior \%GC e IMC ao final do processo da internação ${ }^{9,21}$, porém não de massa magra $^{21}$. Adicionalmente, o estudo de Wilhelm et al. ${ }^{9}$, no qual a maioria dos usuários ao se internarem apresentavam estado nutricional adequado (75\%), seguido de sobrepeso (17,5\%) e apenas 5\% em algum grau de desnutrição, mostrou redução do estado de eutrofia para $50 \%$ e aumento nos percentuais de sobrepeso para $47,6 \%$, além da migração de classificação do estado nutricional dos desnutridos para a eutrofia. É interessante observar que, no estudo de Balbinot et al. ${ }^{20}$, apesar de 
percentual de gordura corporal abaixo do preconizado e de IMC em eutrofia à internação, os pacientes apresentavam relação cintura-quadril indicativa de excesso de gordura abdominal, ao que os autores apontam como fator de risco no dependente de crack, uma vez que está associado à doença cardiovascular e morbimortalidade.

Ferreira et al. ${ }^{22}$ contemporizam resultados de outros autores mostrando IMC eutrófico ${ }^{4,8}$ na maioria dos indivíduos em abstinência em comunidade terapêutica, sugerindo que esses estudos envolvem indivíduos cuja autonomia relativa a opções alimentares é relativamente restrita, pela oferta de alimentação-padrão. Apesar da lógica argumentação, no presente estudo observamos elevada frequência de indivíduos em sobrepeso ou obesidade segundo o IMC. Por outro lado, em acompanhamento ambulatorial de indivíduos em recuperação de dependência química, nos quais se pressupõe certa autonomia nas opções alimentares, foram descritos cerca de $88 \%$ de excesso de peso segundo o IMC, o que também se refletiu na medida da circunferência da cintura, configurando risco cardiovascular aumentado em $44 \%$ e muito aumentado em $24 \%$ deles $^{22}$, sendo que $72 \%$ referiam aumento de consumo alimentar no período de abstinência, com preferência por alimentos específicos (pão francês, sucos artificiais, refrigerantes, embutidos com alto teor de gordura e balas e chicletes) em cerca de $48 \%$ dos casos e elevada frequência de consumo de alimentos ultraprocessados. No presente estudo, mais da metade dos indivíduos acompanhados em CAPS igualmente apresentavam elevado percentual de gordura corporal. Uma possível explicação para nossos achados, tanto nos indivíduos em comunidade terapêutica quanto acompanhados em CAPS, é a fissura sentida na abstinência do $\mathrm{crack}^{23}$. Entre outras estratégias comportamentais e farmacológicas, comer para o alívio da fissura por crack foi citado por usuários, que relataram comer compulsivamente e geralmente carboidratos ${ }^{23}$. No estudo de Ferreira et al. ${ }^{22}, 48 \%$ dos indivíduos em tratamento para dependência química relataram preferência por algum tipo de alimento; destes, $75 \%$ relataram doces e chocolates. O estudo de Teo et al. ${ }^{4}$ encontrou $45,5 \%$ de excesso de peso em indivíduos em comunidade terapêutica em tratamento para dependência química, mostrando que a alimentação na instituição pode contribuir para o excesso de peso, uma vez que foram evidenciados desequilíbrios entre os grupos alimentares, elevada oferta de frituras e de carboidratos complexos. 
No presente estudo, resultados qualitativos do consumo alimentar não parecem por si só justificar as diferenças no estado nutricional entre os que estavam em tratamento para a dependência química e os usuários sem tratamento. Acreditase mais que variáveis quantitativas de consumo diferenciadas entre os grupos em tratamento e sem tratamento, entre outros aspectos, possam ter influenciado o estado nutricional. Essa constatação deriva da observação de que o consumo diário de doces, de carnes e de gorduras e baixa frequência de consumo de frutas e vegetais foram observadas tanto no grupo sem tratamento quanto em frequentadores de CAPS. Aliado a isso, os grupos alimentares contendo amidos, as carnes, leite e gorduras eram oferecidos diariamente aos usuários internados em Comunidade Terapêutica, em contraposição a vegetais e doces que eram apenas oferecidos cerca de três vezes na semana, sendo frutas raramente oferecidas.

Os resultados apresentados sugerem modificação na composição corporal dos indivíduos que passam por um tratamento para a desintoxicação do consumo de crack. Entretanto essa modificação não é necessariamente produtiva para a saúde geral e qualidade de vida do indivíduo, fenômeno que deve ser levado em consideração na construção do plano terapêutico. Embora as diversas estratégias criadas pelo próprio usuário para lidar com a sua fissura possam ser importantes ${ }^{23}$, no caso específico da compensação na abstinência do crack por meio da alimentação, a equipe multiprofissional deve estar atenta aos seus eventuais efeitos sobre a saúde. Entretanto essa modificação não é necessariamente produtiva para a saúde geral e qualidade de vida do indivíduo, fenômeno que deve ser levado em consideração na construção do plano terapêutico. Embora as diversas estratégias criadas pelo próprio usuário para lidar com a sua fissura possam ser importantes ${ }^{23}$, no caso específico da compensação na abstinência do crack por meio da alimentação a equipe multiprofissional deve estar atenta aos seus eventuais efeitos sobre a saúde.

Constituem-se limitações deste estudo o fato de na comunidade terapêutica a oferta alimentar ser padrão, restringindo assim maiores comparativos de consumo alimentar. Além disso, o fato de não ter sido avaliado o uso de medicações cujos efeitos fisiológicos poderiam influir nos resultados, especialmente quanto ao percentual de gordura corporal. O delineamento transversal do estudo limita, adicionalmente, a possibilidade de estabelecer relação de causa/efeito entre o estado 
Artigo Original

Atenção à Saúde

nutricional atual e o consumo alimentar, fatores que podem sofrer impactos também da atividade física, não avaliada neste estudo.

\section{CONCLUSÕES}

Apesar de frequência elevada de usuários de crack classificados como eutróficos segundo o IMC, o fato de estar ou não em tratamento e/ou abstinência da droga aponta para conformações diferenciadas no estado nutricional, com elevada prevalência de indivíduos em tratamento em sobrepeso/obesidade, em contraposição aos usuários sem tratamento. Este aspecto é reiterado pela frequência de usuários sem tratamento com percentuais de GC abaixo da média e alta frequência de indivíduos com percentuais acima da média e/ou risco de doenças ou desordens ligadas à obesidade, nos grupos em tratamento para a dependência química. Práticas alimentares no que se refere à qualidade da alimentação não parecem ser substancialmente diferenciadas entre usuários em tratamento ou não. Os resultados apontam para a importância de vigilância do estado nutricional em ambas as situações: déficit ou sobrepeso/obesidade.

\section{REFERÊNCIAS}

1. II Levantamento Nacional de Álcool e Drogas (LENAD) - 2012. Ronaldo Laranjeira (Supervisão) [et al.], São Paulo: Instituto Nacional de Ciência e Tecnologia para Políticas Públicas de Álcool e Outras Drogas (INPAD), UNIFESP. 2014.

2. Rodrigues VS, Horta RL, Szupszynski KP, Souza MC, Oliveira M. Revisão sistemática sobre tratamentos psicológicos para problemas relacionados ao crack. J bras psiquiatr. 2013;62(3):208-16.

3. Souza J, Kantorski LP. A rede social de indivíduos sob tratamento em um CAPSad: o ecomapa como recurso. Rev esc enferm USP. 2009;43(2)373-83.

4. Teo CPA, Baldissera L, Rech FRF. Adequação da alimentação ao perfil dos dependentes químicos em uma comunidade terapêutica: um estudo de caso. Rev. eletrônica saúde mental álcool drog. 2011;7(3):119-25.

5. Gonçalves VM, Pedroso R, Dos Santos AM, Diemen LV, Pechansky F. Following up crack users after hospital discharge using record linkage methodology: an alternative to find hidden populations. Biomed res int. 2015;2015:973857. doi: $10.1155 / 2015 / 973857$ 
6. Brasil. Ministério da Saúde. Secretaria de Atenção à Saúde. DAPES. Coordenação-Geral de Saúde Mental, Álcool e Outras Drogas. Saúde Mental no SUS: as novas fronteiras da Reforma Psiquiátrica. Relatório de Gestão 2007-2010. Ministério da Saúde: Brasília. Janeiro de 2011.

7. Nasi C, de Oliveira GC, Lacchini AJ, Schneider JF, de Pinho LB. Tecnologias de cuidado em saúde mental para o atendimento ao usuário de crack. Rev. gaúcha enferm. 2015;36(1):92-7.

8. Oliveira ERN, Marin IC, Ferruzzi L, Tenório MFS, Trindade E. Avaliação dos hábitos alimentares e dos dados antropométricos de dependentes químicos. Arq cienc saude Unipar. 2005;9(2):91-6.

9. Willhelm FF, Escobar M, Perry IDS. Alterações na composição corporal e em parâmetros antropométricos de dependentes de crack internados em unidade de adição. J bras psiquiatr. 2013;62(3):183-90.

10. Lizasoian I, Moro MA, Lorenzo, P. Cocaína: aspectos farmacológicos. Adicciones. 2002;14(1):57-64.

11. World Health Organization. Obesity: preventing and managing the total epidemic. Report of a WHO Consultation Group. Geneva: WHO; 1997.

12. Heyward VH, Stolarczyk LM. Fundamentos da Composição Corporal. In: Avaliação da Composição Corporal Aplicada. São Paulo: Manole, 2000.

13. Fisberg RM, Martini LA, Slater B. Métodos de inquéritos alimentares. In: Fisberg RM, Slater B, Marchioni DML, Martini LA. Inquéritos alimentares: métodos e bases científicos. São Paulo: Manole; 2005.

14. da Silva ALMA, Moreno ACCM, Neves LA, de Araújo EC, Frazão IS. Epidemiological profile of crack users in Psychosocial Care Center for Alcohol and Other Drug Users (caps ad). Rev enferm UFPE. 2011;5(spe):2635-43.

15. Sanchez ZVM, Nappo AS. Sequência de drogas consumidas por usuários de crack e fatores interferentes. Rev. saúde pública. 2002;36(4):420-30.

16. Oliveira LG, Nappo SA. Crack-cocaine in São Paulo: accessibility, market strategies and ways of use. Rev psiq clín. 2008;35(6):212-18.

17. Dunn J, Laranjeira R, Silveira DX, Formigoni MLOS, Ferri CP. Crack cocaine: an increase in use among patients attending clinics in Sao Paulo: 1990-1993. Subst use misuse. 1996;31:519-27.

18. Guimarães CF, Santos DVV, Freitas RC, Araújo RB. Perfil do usuário de crack e fatores relacionados à criminalidade em unidade de internação de desintoxicação no Hospital Psiquiátrico São Pedro de Porto Alegre (RS). Rev psiquatr RS. 2008;30(2):101-108. 
19. Mariana Etchepare, Ediléia Rejane Dotto, Káthia Abreu Domingues, Elisângela Colp. Perfil de adolescentes usuários de crack e suas consequências metabólicas. Revista da AMRIGS, Porto Alegre. 2011:55(2):140-6.

20. Balbinot AD, Alves GSL, do Amaral Junior AF, Araujo RB. Perfil antropométrico de dependentes de crack hospitalizados para desintoxicação. Revista HCPA 2011;31(3):311-7.

21. Balbinot AD, Alves GSL, Amaral Junior AF, Araujo RB. Associação entre fissura e perfil antropométrico em dependentes de crack. $\mathrm{J}$ bras psiquiatr. 2011;60(3):205-9.

22. Ferreira IB, Paiva CB, Narvaez JCM,Bosa VL. Estado nutricional e hábitos alimentares de dependentes químicos em tratamento ambulatorial. $J$ bras psiquiatr. 2015;64(2):146-53.

23. Chaves TV, Sanchez ZM, Ribeiro LA, Nappo SA. Fissura por crack: comportamentos e estratégias de controle de usuários e exusuários. Rev saúde pública. 2011;45(6):1168-75. 